This is the peer reviewed version of the following article: Stewart, K. M. and Penlidis, A. (2016), Designing Polymeric Sensing Materials for Analyte Detection and Related Mechanisms. Macromol. Symp., 360: 123-132, which has been published in final form at https://doi.org/10.1002/

masy.201500109. This article may be used for non-commercial purposes in accordance with Wiley Terms and Conditions for Self-Archiving.

\title{
Designing Polymeric Sensing Materials for Analyte Detection and Related Mechanisms
}

\author{
Katherine M. E. Stewart, Alexander Penlidis*
}

Institute for Polymer Research, University of Waterloo, 200 University Avenue

West, Waterloo, Ontario, N2L 3G1, Canada; E-mail: Penlidis@ uwaterloo.ca

\begin{abstract}
Summary: A systematic approach is used to design and tailor sensing materials for targeted analytes and specific applications. An example is used to demonstrate how potential sensing materials can be designed based on the chemical nature of both the target analyte and the sensing material, and thus predominant sensing mechanisms by which the two interact. The example analyte is a small, polar molecule able to hydrogen bond; therefore, a sensing material that targets the analyte should have polymer chains that pack tightly together, be polar, and be able to hydrogen bond. Any metal oxide dopants should be able to coordinate to both the target analyte and the polymer. Polyaniline and poly (o-anisidine), along with nickel oxide and zinc oxide, are chosen as potential sensing materials and subsequently evaluated based on their ability to sorb the analyte in question.
\end{abstract}

Keywords: gas sensor; sensing mechanisms; polyaniline; poly (o-anisidine)

\section{Introduction}

Sensing materials are the "heart" of a sensor since the sensing material is what interacts with the analyte(s) to be detected. By changing the sensing material, a sensor can be used to target different analytes. Multiple sensing materials can also be used on a single sensor array to detect multiple analytes $^{[1]}$ or create a more selective sensor for a specific analyte. ${ }^{[2]}$

Current methods of developing sensing materials rely on a materials science approach. Generally, a new material is made and characterized using various techniques, including (usually) scanning electron microscopy (SEM), transmission electron spectroscopy (TEM), and X-ray diffraction 
(XRD). ${ }^{[3,4]}$ The material is then deposited onto a commercially available sensor and typically evaluated using a single gas analyte. ${ }^{[5]}$ This method of developing new sensing materials can be very time consuming and costly.

A more direct approach of designing sensing materials based on how they interact with the target analyte is presented herein. Analytes interact with sensing materials through multiple sensing mechanisms, simultaneously; however, some sensing mechanisms will dominate depending on the chemical nature of both the sensing material and analyte. By understanding the chemical nature of an analyte and the most likely dominant sensing mechanism, it is possible to select potential polymeric sensing materials that will target the analyte. These polymers can be further tailored by modifying the main backbone (by introducing functional groups and side chains), as well as incorporating dopants such as metals and metal oxides.

\section{Sensing Mechanisms}

Sensing mechanisms describe how an analyte and a sensing material interact. Aspects may include what attracts/repels the analyte, as well as how the analyte sorbs onto the sensing material. An analyte may either adsorb onto the surface of a sensing material (at a sensing site) and weakly bind through electrostatic interactions, or absorb into the sensing material, by diffusing into the interstitial spaces created within the packed polymeric chains of a sensing material.

By examining the chemical nature of both the analyte and sensing material, it is possible to determine the predominant mechanism(s) by which the analyte and sensing material will likely interact. However, multiple mechanisms are always at play and they may counteract one another. For example, polarity and Lewis acid-base mechanisms are attractive in nature, whereas steric hindrance is repulsive. The attractive and repulsive forces will counteract one another; however, 
one mechanism may dominate (even if slightly), which results in the analyte sorbing or not sorbing onto the sensing material. Understanding potential mechanisms allows them to be identified given an analyte's chemical composition, and thus, allows sensing materials to be designed to target specific analytes.

\subsection{Polarity and Hydrogen Bonding}

Based on the electronegativity of the atoms in a molecule and its structure, a molecule with covalent bonds is either polar or non-polar. Since all volatile organic compounds (VOCs) are covalently bonded, they can all be classified by their polarity. The degree of polarity is measured using a dipole or the dipole moment (which is the overall dipole for the entire molecule). Symmetric molecules, such as carbon tetrachloride $\left(\mathrm{CCl}_{4}\right)$, are all non-polar and have a dipole moment of $0 \mathrm{D}$, despite possibly having a dipole between the atoms in the molecule. As an example, $\mathrm{CCl}_{4}$ has dipoles between the carbon and chlorine due to the difference in electronegativities of the atoms: carbon (2.55) and chlorine (3.16). This is because the four chlorine atoms are equally spaced around the carbon in a tetrahedral configuration and thus, the dipoles cancel each other out, resulting in a net dipole moment of $0 \mathrm{D}$.

A molecule is considered non-polar if the atoms have similar electronegativities such as carbon (2.55) and hydrogen (2.20). Therefore, all hydrocarbons (which contain only carbon and hydrogen) are all considered non-polar.

Polar molecules have varying degrees of polarity; the larger the dipole moment, the more polar a molecule is. The dipole is affected by the atoms within a molecule. The more electronegative atoms (such as oxygen and nitrogen) draw electron density towards themselves, resulting in those atoms becoming even more negative. This leaves the atoms from which the electron density was 
pulled more positive. Since dipoles represent a charge distribution across a molecule, polar molecules are attracted to one another, through electrostatic forces. Therefore, when the target analyte is polar, then a polar sensing material will attract the analyte. As with all electrostatic interactions, the more polar the molecules (both analyte and sensing material), the stronger the attraction between them.

A special case of polarity is hydrogen bonding. This is the result of a very large charge distribution between a hydrogen (2.1) and either a nitrogen (3.0), oxygen (3.5), or fluorine (4.0) atom. The large charge distribution creates a large dipole between the two atoms, which through electrostatic attraction, are able to create a weak bond with another molecule that also has a large dipole, known as a hydrogen bond. This bond is considered a physical bond, and can be easily broken. Therefore, an analyte that bonds to a sensing material through a hydrogen bond may be removed fairly easily, allowing the sensing material to be reused.

\subsection{Lewis Acid-Base Interactions}

Lewis acid-base interactions occur when a Lewis base seeks out a Lewis acid. A Lewis base is defined as a molecule that donates a pair of electrons and a Lewis acid is a molecule that accepts a pair of electrons. Lewis bases include molecules that contain oxygen and nitrogen, both of which contain at least one lone pair of electrons (nitrogen has one pair and oxygen has two pairs), hence capable of acting as a Lewis base. Lewis acids include molecules that have atoms that have a lower electron density due to the charge distribution across the molecule. For example, the carbonyl carbon $(\mathrm{C}=\mathrm{O})$ in acetone is slightly positive due to the double bonded oxygen pulling electron density away from the carbon. Therefore, the carbonyl carbon acts as a Lewis acid. Since 
acetone contains a double bonded oxygen, it can also act as a Lewis base due to the lone pairs of electrons on the oxygen.

Lewis acid-base interactions are attractive electrostatic forces. The lone pairs of electrons on an atom, that tend to have a slight negative charge due to the atom drawing electron density from the rest of the molecule, are attracted to a slightly positive atom in another molecule. The Lewis base wants to share its electron density and the Lewis acid wants to gain more. While it is possible for Lewis acid-base interactions to produce a chemical bond, as a sensing mechanism, this does not generally occur because there is not sufficient energy available to the molecules to form a chemical bond. Therefore, the interaction between a Lewis acid and base is weak and can be reversed. Thus, the analyte can be removed from the sensing material and the sensing material can be reused.

\subsection{Metal Coordination}

Metal coordination only exists when a metal is present. Metals and metal oxides can be added in small amounts (this is referred to as doping) to a polymeric sensing material to improve various properties, including sensitivity, selectivity, mechanical, and/or electrical. ${ }^{[6,7]}$ Through electrostatic forces, a metal is able to attract and coordinate to multiple ligands (molecules). For example, zinc $(\mathrm{Zn})$ tends to have a coordination number of 4 , which means it can coordinate with 4 ligands. Zn can coordinate with the sensing material in at least one place, but possibly more, leaving up to three ligand sites for the analyte with which to coordinate.

It should be noted that not all molecules are able to coordinate all metals. The biggest factor in coordination occurs once the molecules are close enough to the metal to coordinate. If the molecule has a lone pair of electrons in an s- or p-orbital in a similar energy level as an empty dorbital in the metal, then the lone pair of electrons is able to share the electrons with the d-orbital 
of the metal and thus, creates a covalent, coordination bond. Without delving too much into molecular orbital theory, it is possible to identify metal and metal oxides that will likely coordinate with the target molecule (without knowing the molecular orbital energies). This is accomplished by choosing metals and metal oxides that are used as catalysts for breaking down the target analyte (see Section 3.3.2: Metal Oxide Dopants for more information).

\section{$2.4 \quad$ Steric Hindrance}

The previous three mechanisms were all based on attractive forces that brought the analyte towards the sensing material and then caused the analyte to bind to the material. Steric hindrance, on the other hand, acts as a repulsive force. It is caused by the electronic repulsion of the electrons in molecules. All molecules are surrounded by a cloud of electrons that repel the molecule in question from other molecules. The bulkier a molecule, the larger the electronic cloud that surrounds it and, therefore, the harder it is for that molecule to come near another molecule. In the case of analytes, bulkier analytes are repelled more from a sensing material and are less able to fit into the interstitial spaces of a sensing material. Therefore, smaller, less bulky analytes (such as ethanol or formaldehyde) are able to interact with a sensing material more easily than larger, bulkier analytes (such as benzene).

Steric hindrance can be used to improve selectivity of a sensing material. By choosing a polymeric sensing material that packs closely together, and thus creates small interstitial spaces between the chains, larger and bulkier analytes are less able to diffuse into the interstitial spaces due to steric 
hindrance (or repulsion). This allows the sensing material to act as a filter, filtering out larger analytes, and thus improving the selectivity of a material towards the smaller target analyte.

Steric hindrance can also be used positively, in that adding bulkier or longer side chains to a polymer reduces the ability of the polymer to pack as closely. This increases the size of the interstitial spaces and thus allows analytes to diffuse more easily into the sensing material. While this may reduce the selectivity of the sensing material, it may improve its sensitivity.

\section{Selecting Sensing Materials}

A systematic approach is used to select and design sensing materials for specific applications. Sensing materials can be designed and selected based on the sensing mechanisms described, as well as other requirements needed for sensor applications. Initially, looking at the target analyte from a chemical perspective gives an idea of how the analyte may interact with a sensing material. For example, a polar analyte will be attracted to a polar sensing material. This will give a starting point for types of polymers that can be used, albeit, a large list.

Secondly, by looking at the requirements for the final application, the list of potential sensing materials can be narrowed further. For example, a higher temperature application will require a higher glass transition temperature because the polymeric sensing material should not "soften" while in use because this would render the sensor useless.

Finally, it is important to consider the type of sensor used. A resistive sensor requires a conductive sensing material, whereas a conductive sensing material on a capacitive sensor can short circuit the sensor, rendering the sensor useless.

By considering all of these aspects, it is possible to narrow down the list of all possible polymeric materials to a more manageable list of polymers. Dopants may also be chosen to improve the 
properties of the sensing material. From here, sensing materials are ranked based on their potential to interact with the analyte, and the highest ranked polymers (with dopants) are then synthesized and evaluated.

To illustrate this idea, sensing materials will be selected for a sensor that targets ethanol. The sensor will be used above room temperature, and require high sensitivity. The sensing material will be placed on some type of MEMS-based sensor, perhaps capacitive or mass-based.

\section{1 $\quad$ Example Target Analyte}

By analyzing the chemical nature of the target molecule, it is possible to narrow down the type of polymers that can be used as potential sensing materials. The target molecule in this case is ethanol. Ethanol is a small $(3.8 \AA)$ molecule with one alcohol $(\mathrm{OH})$ group. The small size of ethanol means that ethanol can diffuse into small interstitial spaces between polymer chains. By using a polymer that packs closely together, only small interstitial spaces are available into which analytes can diffuse. Therefore, larger interferents (other analytes present along with ethanol) may not be able to diffuse into such spaces, resulting in improved selectivity towards ethanol.

The oxygen in the alcohol group on ethanol draws electron density towards itself, creating a dipole. The dipole moment of ethanol is $1.66 \mathrm{D}$ and therefore, it is a polar molecule. The alcohol group on ethanol is also capable of hydrogen bonding due to the charge differential between the oxygen and hydrogen. Because ethanol is a polar molecule and able to hydrogen bond, the polymeric sensing material should also be polar and ideally also capable of hydrogen bonding.

By using this information, potential polymeric sensing materials have been narrowed down to polymers with polar backbones and side chains. These are polymers that contain functional groups including alcohols, amines, carboxylic acids, and amides. Less branching or no branching may 
result in a more compact polymer matrix and thus, tighter packing and smaller interstitial spaces. However, there is a trade off in terms of sensitivity and selectivity with interstitial space size. Also, more functional groups or branching may increase the number of sensing sites within a polymer matrix to which ethanol is able to bind. Thus, larger interstitial spaces with more sensing sites may work as well.

Selecting metals and metal oxide dopants to improve the sensitivity and/or selectivity of the sensing material is done based on the metal oxides that are able to coordinate to the target analyte. While the metal or metal oxide must be able to coordinate to both the analyte and sensing material, the dopants are chosen based on their ability to coordinate to the target analyte. These dopants are chosen based on their catalytic activity towards the analyte. For example, zinc oxide $(\mathrm{ZnO})$ is a common catalyst towards ethanol oxidation. ${ }^{[8]}$ Ethanol is drawn towards and able to coordinate well with $\mathrm{Zn}$.

When designing sensing materials with greater selectivity, one must consider the predominant mechanisms of both the target analyte and its common interferents. For example, ethanol is the target analyte and acetone, methanol, and benzene are common interferents (analytes) that are present with ethanol. Ethanol is polar and able to hydrogen bond; therefore, the sensing material should be able to hydrogen bond or at least be polar in nature. Methanol is also capable of hydrogen bonding and is smaller. Thus, it may be more difficult to separate from ethanol; however, the addition of a metal or metal oxide that is more likely to coordinate with ethanol than methanol, may improve selectivity. Acetone is also polar, but unable to hydrogen bond. Choosing a sensing material that is capable of hydrogen bonding should reduce the response towards acetone. Finally, benzene is non-polar and larger than the other analytes; therefore, it is less likely to produce a signal when tailoring a sensing material towards ethanol. In addition, benzene may be 
excluded somewhat based on its size through steric interactions and smaller pores within a polymeric sensing material.

\subsection{Sensor Application Requirements}

The final application of the sensor will also have some requirements. For example, a sensor must be robust and environmentally stable, and therefore, so must the sensing material. ${ }^{[9]}$ For a reusable sensor, analytes must be easily removed from the sensing material so the sensor can be regenerated and reused. In this case, sensing materials that chemically bind or strongly bind to analytes are not desirable. Therefore, for a reusable ethanol sensor, a sensing material that binds to ethanol using hydrogen bonds would be good because they are weak physical bonds that can be easily broken and thus, the sensing material can be reused.

Temperature is another more specific sensor requirement. For this example, the sensor could be operated at a temperature up to $60^{\circ} \mathrm{C}$. Therefore, the polymeric sensing material must have a glass transition temperature above $60^{\circ} \mathrm{C}$, since the sensing material should not "soften" while in operation. Thus, polymers with lower glass transition temperatures should not be used.

The type of sensor is critical in designing polymeric sensing materials. For example, a conductive type sensor requires a conductive polymer as a sensing material. When an analyte binds with a conductive sensing material, the resistance of the material changes, and that change is measured by the sensor. ${ }^{[10]}$ Similarly, a change in capacitance is measured for a capacitive sensor, which is either caused by a change in dielectric constant or swelling of the sensing material. However, depending on the architecture of the capacitive sensor, a conductive polymer might short circuit the sensor, and thus a conductive polymer cannot be used as a sensing material for that type of 
sensor. ${ }^{[11]}$ For a mass-based sensor, such as a micro-cantilever, a light sensing material is required, such as a polymer instead of a metal or metal oxide. ${ }^{[12]}$

For our example, the type of sensor has not been defined, thus a few sensing materials that have some flexibility in terms of working on different sensors will be discussed. Note that different sensing materials work better on different types of sensors and thus, sensing materials must be evaluated on each type of sensor to determine optimal performance.

\subsection{Potential Sensing Materials}

Based on the requirements listed in Sections 3.1 and 3.2, a potential polymeric sensing material for ethanol should be polar, ideally capable of hydrogen bonding, and have polymeric chains that are able to pack tightly together. The polymer should be environmentally stable and have a glass transition temperature above $60^{\circ} \mathrm{C}$. Since the type of sensor has not been defined in our example, several polymers will be considered. In addition, any metal or metal oxide dopant chosen should be able to coordinate with ethanol.

\subsubsection{Polymers}

Considering all these factors, as well as ease of synthesis, processability, and cost, polyaniline (PANI) was chosen as a potential sensing material for ethanol (see Figure 1). PANI contains an amine group that makes PANI both polar and able to hydrogen bond. It is possible for the hydrogen in the ethanol to bond to the nitrogen in PANI and the hydrogen in PANI to bond to the oxygen in ethanol. Therefore, it is possible to have two ethanol molecules hydrogen bonded to each N-H in PANI. This, however, is limited by the space surrounding the nitrogen. Steric hindrance may limit the access of ethanol to the nitrogen on PANI. ${ }^{[13]}$ 


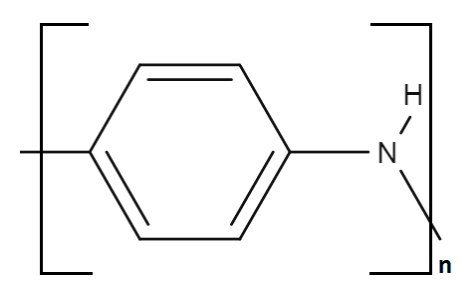

Figure 1. Polyaniline (PANI).

PANI is environmentally stable and despite not having a glass transition temperature, PANI doped with acid has a degradation temperature of $104^{\circ} \mathrm{C}$; however, undoped PANI is stable above $300^{\circ} \mathrm{C} .^{[14]}$ Thus, PANI will remain useable up to $100^{\circ} \mathrm{C}$, well above the requirements needed. PANI is also simple to make, moderately processable, and inexpensive.

Finally, PANI is unique in that it can be made either conductive or nonconductive. By doping PANI with an acid, it becomes conductive. The addition of protons (from the acid) to PANI creates positively charged nitrogen atoms, creating holes along the polymer chain. These holes allow valence electrons to travel along the polymer chain by jumping from one hole to another, thereby making PANI conductive. ${ }^{[15]}$ This unique ability of being either conductive or nonconductive allows flexibility in the type of sensor that can be used.

Using PANI as a basis for a sensing material, it can then be modified (tailored) to potentially improve its sensitivity to ethanol. Adding a methoxy group to PANI, to create poly ( $o$-anisidine) (PoANI), increases the number of sensing sites per monomer unit, since ethanol is able to bind to both the amine and now the oxygen from the methoxy group, assuming no resistance due to steric hindrance (see Figure 2). ${ }^{[16]}$ PoANI will not pack as tightly as PANI; however, this may increase the sensitivity to ethanol by increasing the mobility of ethanol through the polymer chains, despite 
possibly reducing selectivity. These trade-offs are common in sensing material/sensor selection efforts.

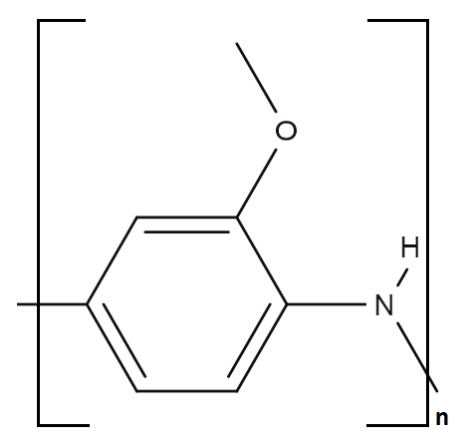

Figure 2. Poly (o-anisidine) (PoANI).

\subsubsection{Metal Oxide Dopants}

When selecting potential metal oxide dopants for sensing materials, it is most important that the metal coordinates with the target analyte. Selecting metal oxides that are used in catalyzing reactions involving the target analyte, ensures that the analyte is attracted to and able to coordinate with the metal. The metal should also be able to coordinate with the polymer, such as $\mathrm{NiO}$ and PANI. ${ }^{[17]}$

Ethanol is commonly degraded using $\mathrm{ZnO} \cdot{ }^{[18]}$ Therefore, ethanol is able to coordinate well with Zn. Similarly, ethanol may also be oxidized using $\mathrm{NiO} \cdot{ }^{[19]}$ Both $\mathrm{ZnO}$ and $\mathrm{NiO}$ (sometimes even both in combination ${ }^{[20]}$ ) are also used as metal oxide sensing materials in many gas sensors. Therefore, $\mathrm{ZnO}$ and $\mathrm{NiO}$ were chosen as potential dopants to be incorporated into both PANI and PoANI.

\section{Experimental Part}




\subsection{Synthesis of Polymeric Materials}

PANI was synthesized by mixing aniline, ammonium persulfate, and, if present, the dopants, in deionized water. $0.39 \mathrm{~mL}$ of aniline (A.C.S. reagent, purity $\geq 99.5 \%$, Sigma-Aldrich, Oakville, Ontario, Canada) was added to $20 \mathrm{~mL}$ of deionized water and then mixed using a sonicator for 30 minutes. This solution was then cooled to $-1^{\circ} \mathrm{C}$ before the addition of a solution containing $1.0 \mathrm{~g}$ of ammonium persulfate (A.C.S. Reagent, Sigma-Aldrich) in $5 \mathrm{~mL}$ of deionized water. The solution was shaken for one minute to ensure thorough mixing. The mixture was subsequently left to react at $-1^{\circ} \mathrm{C}$ for 6 hours. ${ }^{[13]}$ The polymer was filtered out using a funnel and Whatman \#5 filter paper and left overnight. The polymer was then washed with ethanol until the liquid ran clear. Finally, the polymer was scraped into a glass vial for storage under atmospheric conditions.

To obtain the doped polymer, the monomer was polymerized with the dopant suspended in the starting solution. The dopant was added up to $20 \%$ by weight with respect to the monomer, before the solution was initially cooled prior to the addition of the ammonium persulfate. Other than the addition of the dopant, which was nickel oxide $(\mathrm{NiO})$ (particle size $<50 \mathrm{~nm}$, concentration of 99.8\%, from Sigma-Aldrich) or zinc oxide $(\mathrm{ZnO})$ (particle size $<100 \mathrm{~nm}, 50 \mathrm{wt}$ \% in water, from Sigma-Aldrich), the polymerization procedure was the same as described above for PANI without any dopant.

PoANI was prepared in the same manner as PANI, except $o$-anisidine (A.C.S. reagent, purity $\geq 99 \%$, Sigma-Aldrich, Oakville, Ontario, Canada) was used as the monomer instead of aniline. The two polymers were prepared with two different dopants, $\mathrm{NiO}$ and $\mathrm{ZnO}$, at three different concentrations ( $0 \%$ or no dopant, $10 \mathrm{wt} . \%$ and $20 \mathrm{wt} . \%)$. In total, ten different polymeric sensing materials were prepared for initial screening. 
The sensing materials were evaluated using gaseous ethanol in tanks from Praxair (California, USA). The 5 ppm of standard grade gaseous ethanol in nitrogen was used. Nitrogen (5.0 grade, Praxair, Mississauga, Ontario, Canada) was used to purge the sensing materials prior to evaluation using ethanol. The potential polymeric sensing materials were evaluated using the gas test system described in Section 4.2.

\subsection{Experimental Set-up}

To evaluate the potential sensing materials, gas sorption tests were performed. Each sensing material was exposed to $5 \mathrm{ppm}$ of ethanol gas (in a balance of nitrogen) and the amount of ethanol that sorbed onto the sample was measured. The more ethanol that sorbed onto the sensing material, the more sensitive the sensing material was to ethanol. Measurements were conducted at room temperature $\left(22^{\circ} \mathrm{C}\right)$ and slightly above atmospheric pressure $(15 \mathrm{psi})$.

These tests were conducted using a specifically designed test system. Ethanol gas (5 ppm from a cylinder) passed through an MSK RS-485 mass flow controller (MFC) that limited the flow rate to $200 \mathrm{sccm}$, then through a passive mixer. This was followed by an MKS 640A pressure controller that kept the pressure slightly above atmospheric pressure (15 psi) and an MKS 1179A flow meter that ensured $200 \mathrm{sccm}$ flow rate was maintained. The gas then passed through a $100 \mathrm{~mL}$ glass flask that held the polymeric sensing material (sample) and subsequently into a specialized Varian 450 gas chromatograph (GC) with a photon discharge helium ionization detector (PDHID), capable of measuring down to the ppb level for different compounds (see Figure 3). 


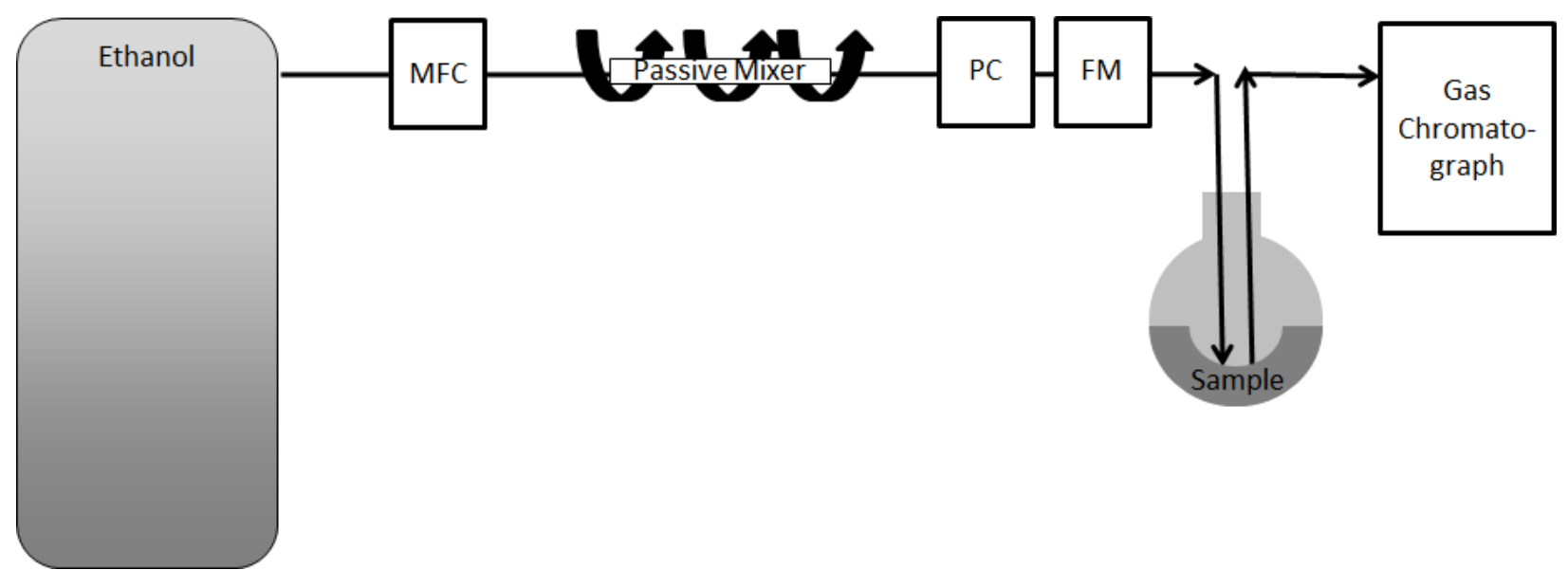

Figure 3. Schematic of the test system, where MFC, PC, and FM are mass flow controller, pressure controller, and flow meter, respectively.

A baseline was measured using an empty $100 \mathrm{~mL}$ flask, which was used to determine the initial concentration of the ethanol. A flask that contained $0.120 \mathrm{~g}$ of the sensing material was purged using dry nitrogen gas for 30 minutes and subsequently replaced the empty flask. The flask containing the sensing material was left on-line for 30 minutes, while $5 \mathrm{ppm}$ of ethanol passed over it. This ensured that sorption equilibrium was reached. The amount of ethanol that did not sorb onto the sensing material continued downstream and was measured by the GC. By subtracting the amount of ethanol that did not sorb, the amount of ethanol that sorbed onto the sensing material was obtained. ${ }^{[21]}$

\section{Results and Discussion}

Since deposition onto sensors can be costly and time-consuming, potential sensing materials are first evaluated based on the amount of ethanol sorbed. Each sensing material was exposed to 5 ppm of ethanol and the amount of ethanol that sorbed onto the sensing material was measured using a GC. Three independent replicates were performed (shown by open circles) for each sensing material and appear in Figure 4, along with the average (shown by $\mathrm{x}$ ). 


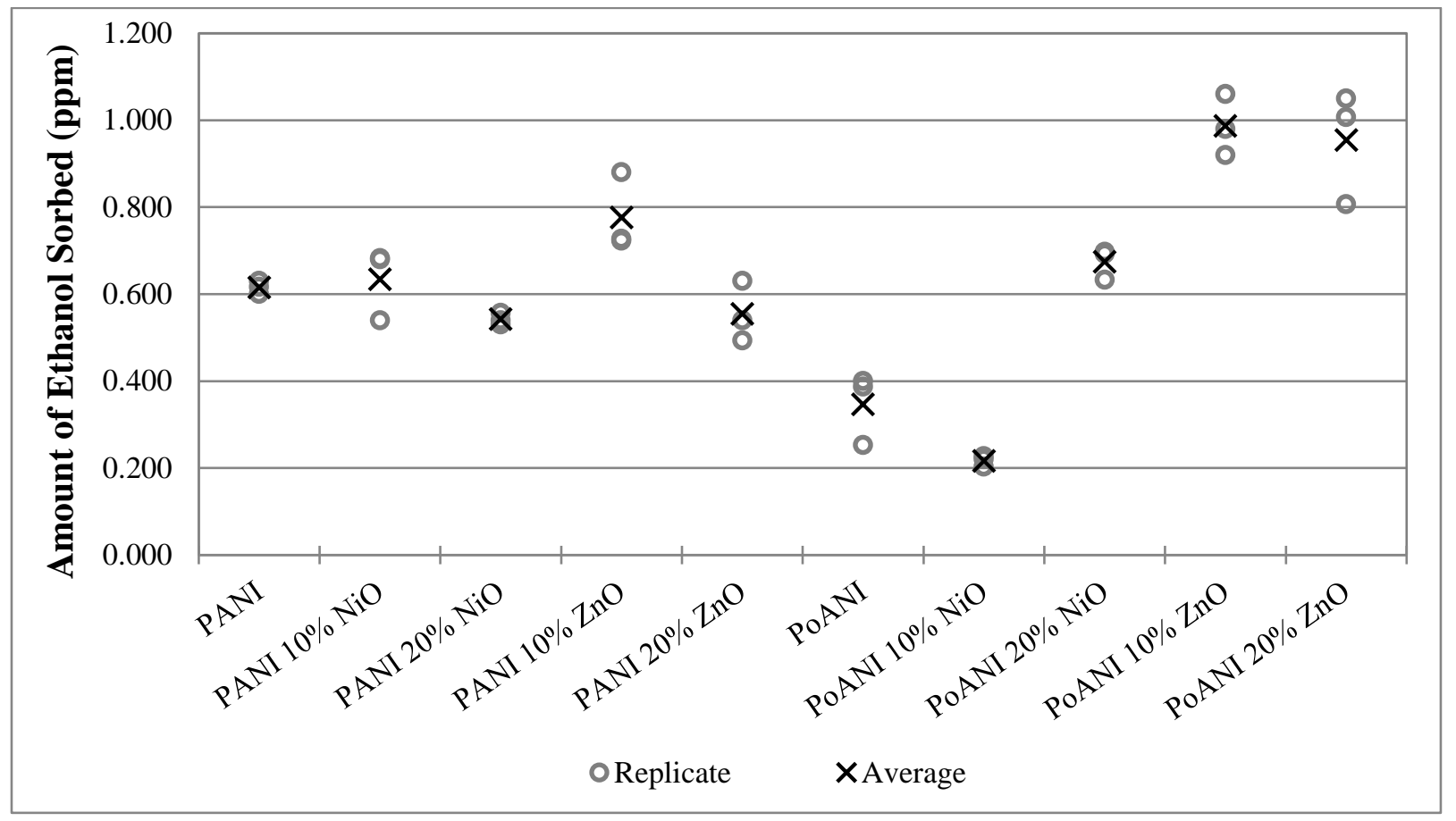

Figure 4. Sorption for each sensing material when exposed to $5 \mathrm{ppm}$ ethanol.

Based on the results shown in Figure 4, PoANI doped with $\mathrm{ZnO}$ should be evaluated further. Since different sensing materials behave differently on different types of sensors, PANI $10 \%$ ZnO should also be evaluated on different types of sensors because it performed moderately well. Since PoANI $10 \% \mathrm{ZnO}$ and PoANI $20 \% \mathrm{ZnO}$ sorbed similar amounts of ethanol, initially, PoANI $10 \% \mathrm{ZnO}$ should be deposited onto a sensor for further evaluation. This is because PoANI $10 \% \mathrm{ZnO}$ is less expensive than PoANI $20 \% \mathrm{ZnO}$, since $\mathrm{ZnO}$ nanoparticles are more expensive than the polymeric material. PoANI $20 \% \mathrm{ZnO}$ could also be evaluated on different sensors. It is possible that the additional $\mathrm{ZnO}$ may improve the sensitivity of a sensor, which cannot be determined from sorption tests. For example, on a resistive type sensor, the additional $\mathrm{ZnO}$, in the PoANI $20 \% \mathrm{ZnO}$, may improve the conductivity of the polymeric material and thus a larger change in resistance may be measured when ethanol interacts with the sensing material; however, the opposite may also be 
true. Therefore, it is important to evaluate different sensing materials carefully on different sensors to determine which sensor and sensing material combinations work best.

It is important to note that the nonconductive form of PANI (emeraldine salt) was synthesized and used in these evaluations. Therefore, to evaluate these sensing materials on resistive type sensors, these materials must be doped with acid. This is likely to affect the sensing properties of the sensing materials; however, since the addition of acid creates positive charges on some nitrogen atoms, it is likely that doping the polymeric materials with acid will improve their affinity to ethanol. The positive charge on the nitrogens should more strongly attract ethanol than the weaker dipole of the uncharged polymer. The additional hydrogen bound to the nitrogen on the amine of PANI (and PoANI), would reduce the packing density of the polymer chains slightly since $\mathrm{N}-\mathrm{H}_{2}$ takes up more room than $\mathrm{N}-\mathrm{H}$, thus increasing the interstitial spacing within the polymer chains. This should also improve the mobility of ethanol through the polymer matrix and thus, the sensitivity towards ethanol.

\section{Concluding Remarks}

Instead of taking a materials approach and starting with a material, characterizing it, and then evaluating it as a sensing material, a targeted approach was used. Based on the chemical nature of the target analyte and the end-use application, two polymeric materials were synthesized with two different dopants at varying concentrations. The sensing materials were designed by determining the predominant mechanisms by which the target analyte was likely to interact with the sensing material.

In total, only ten sensing materials were evaluated based on their ability to sorb ethanol, where the materials that sorbed the most were the most promising. Out of the ten sensing materials, three 
sensing materials (PoANI 10\% and 20\% $\mathrm{ZnO}$ and PANI $10 \% \mathrm{ZnO}$ ) should be deposited onto different types of sensors for further evaluation.

This systematic approach considerably reduces the amount of experimental work needed to find appropriate sensing materials for a targeted application. By designing and tailoring polymeric sensing materials based on sensing mechanisms, the cost and time associated with current approaches is reduced. In addition, evaluating sensing materials based on their ability to interact with the target analyte, further reduction in cost and time is achieved since only the most promising sensing materials need to be deposited onto sensors for further evaluation.

Acknowledgements: The authors are grateful for financial support from the Natural Sciences and Engineering Research Council (NSERC) of Canada, the Canada Research (CRC) program, and AUTO21.

[1] A. K. Srivastava. Sens. Act. B. 2003, 96, 24.

[2] N. Han, Y. Tian, X. Wu, and Y. Chen. Sens. Act. B. 2009, 138, 228.

[3] T. Itoh, I. Matsubara, W. Shin, N. Izu. Mater. Lett. 2007, 61, 4031.

[4] E. Park, O. S. Kwon, S. J. Park, J. S. Lee, S. You, J. Jang. J. Mater. Chem. 2012, 22, 1521.

[5] R. A. G. Rañola, I. Conina, F. B. Sevilla, M. Ferroni, L. Sangaletti, G. Sbervegileri, E. Comini, presented at AISEM XVIII, Trento, Italy, Feb. 3-5, 2015.

[6] X. Chen, J. Sun, J. Shen. Langmuir. 2009, 25, 3316.

[7] J. A. Dirksen, K. Duval, T. A. Ring. Sens. Act. B. 2001, 8, 106.

[8] J. Xu, J. Han, Y. Zhang, Y. A. Sun, B. Xie. Sens. Act. B. 2008, 132, 334.

[9] S. Virji, J. Huang, R. B. Kaner, B. H. Weiller. Nano Lett. 2004, 4, 491.

[10] D. Nicolas-Debarnot, F. Poncin-Epaillard. Anal. Chim. Acta. 2003, 475, 1.

[11] W. T. Chen, K. M. E. Stewart, R. Mansour, A. Penlidis. Sens. Act. A. 2015, 230, 63.

[12] M. E. Khater, M. Al-Ghamdi, S. Park, K. M. E. Stewart, E. M. Abdel-Rahman, A. Penlidis, A. H. Nayfeh, A K. S. Abdel-Aziz, M. Basha. J. Micromech. Microeng. 2014, 24, 065007.

[13] K. M. E. Stewart, N. T. McManus, E. Abdel-Rahman, A. Penlidis. J. Macromol. Sci., Pure Appl. Chem. 2012, 49, 1.

[14] G. Song, J. Han, and R. Guo. Synthetic Met. 2007, 157, 170.

[15] A. L. Kukla, M. Shirshov, S. A. Piletsky. Sens. Act. B. 1996, 37, 135.

[16] A. A. Athawale, M. V. Kulkarni. Sens. Act. B. 2000, 67, 173.

[17] J. Han, G. Song, R. Guo. J. Polym. Sci. Part A: Polym. Chem. 2006, 44, 4229.

[18] J. M. Vohs, M. A. Barteau. Suf. Sci. 1989, 221, 590.

[19] D. K. Liguras, K. Goundani, X. E. Verykios. J. Power Sources 2004, 130, 30.

[20] C. W. Na, H.-S. Woo, J.-H. Lee. RSC Advances. 2012, 2, 414. 
[21] K. M. E. Stewart, A. Penlidis. Macromol. Symp. (PRE VIII) 2013, 324, 11. 